\title{
Hipersensibilidade aos $\beta$-lactâmicos e outros antibióticos. Bases biológicas
}

\author{
Hypersensibility to $\beta$-lactams and other antibiotics. Biological Basis \\ Hipersensibilidad a los $\beta$-lactámicos y otros antibióticos. Bases Biológicas \\ Maria Isabela Lopes GANDOLFO ${ }^{1}$ \\ Naara Gabriela MONTEIRO ${ }^{1}$ \\ Marcella Santos JANUZZI ${ }^{1}$ \\ Ana Carolina Zucon BACELAR ${ }^{1}$ \\ Marielise Mundin Abrão RODRIGUES ${ }^{1}$ \\ Ana Cláudia OKAMOTO ${ }^{1}$ \\ Christiane Marie SCHWEITZER ${ }^{2}$ \\ Elerson GAETTI-JARDIM JÚNIOR ${ }^{1}$ \\ ${ }^{I}$ Departamento de Patologia e Propedêutica Clínica, Faculdade de Odontologia, UNESP Univ. Estadual Paulista 16015-050, Araçatuba-SP, Brasil. \\ ${ }^{2}$ Departamento de Matemática, Faculdade de Engenharia, UNESP Univ. Universidade Estadual Paulista 15385-000, Ilha Solteira-SP, Brasil.
}

\begin{abstract}
Resumo
Este estudo teve como objetivo avaliar a ocorrência de alergias aos antibióticos e antimicrobianos comumente utilizados em medicina e odontologia, discutindo os mecanismos envolvidos nas reações de hipersensibilidade. Pela literatura verificou-se que a maioria das alergias foi associada com o uso frequente de tais compostos e depende de medicamentos haptenização. A hipersensibilidade pode ser classificada em reacções immediadas e aceleradas, associadas com respostas humorais, ou mediadas por linfócitos T que reconhecem o antigénio apresentado por células apresentadoras de antigenos e liberam citocinas, resultando no recrutamento de diferentes tipos celulare, desencadeando a inflamação do tecido. Além disso, as declarações de 386 pacientes odontológicos foram analisadas e ocorrência de alergias aos antibióticos foram relatadas por $6,2 \%$ dos pacientes. As reacções às penicilinas foram predominantes $(4,7 \%)$, seguidas da sulfanilamida $(1,0 \%)$ e das cefalosporinas $(0,5 \%)$. A sintomatologia predominante foi essencialmente cutânea. Também foram analisadas as consequências, diagnóstico e prevenção das alergias aos antimicrobianos. Este estudo concluiu que os $\beta$-lactâmicos foram as drogas mais alergênicas e a grande maioria das reações imunes foram desencadeadas contra eles. O tratamento de dessensibilização deve ser considerado a última abordagem clínica devido aos seus riscos para os pacientes.

Descritores: Hipersensibilidade a Drogas; Antibacterianos; Alergia e Imunologia.
\end{abstract}

\begin{abstract}
This study aimed to evaluate the occurrence of allergies to antibiotics and antimicrobials commonly used in medicine and dentistry, discussing the mechanisms involved in hypersensitivity reactions. In literature, it was verified that most allergies were associated with incremental use of such chemical compounds and depended on the drugs haptenation. Hypersensitivity can be classified into ready mediated and accelerated reactions, associated with humoral responses, or mediated by $\mathrm{T}$ lymphocytes that recognize antigen displayed by antigen-presenting cells and release cytokines, resulting in recruitment of several cell types and also trigger a tissue inflammation. In addition, declarations of 386 dental patients were analyzed and allergies were reported by $6.2 \%$ of patients. Reactions to penicillins predominated $(4.7 \%)$, followed by sulfanilamide $(1.0 \%)$ and cephalosporins $(0.5 \%)$. Predominant symptomatology was essentially cutaneous. The consequences, diagnosis and prevention of drug allergy were also discussed. This study concluded that $\beta$-lactams were the most allergenic drugs and the vast majority of immune reactions were triggered against them. Desensitizing treatment must be considered the last clinical approach due to its risks for patients.
\end{abstract}

Descriptors: Drug Hypersensitivity; Anti-Bacterial Agents; Allergy And Immunology.

\section{Resumen}

Este estudio tuvo como objetivo evaluar la aparición de alergias a los antibióticos y antimicrobianos de uso común en la medicina y la odontología, y discutir los mecanismos implicados en las reacciones de hipersensibilidad. Por la literatura se encontró que la mayoría de las alergias se asocia con el uso frecuente de tales compuestos y depende de haptenación. Hipersensibilidad puede clasificarse en immediadas y reacciones aceleradas asociada con respuestas humorales, o mediadas por linfocitos $\mathrm{T}$ que reconocen el antígeno presentado por las células presentadoras de antígeno y liberan citoquinas, lo que resulta en el reclutamiento de diferentes tipos celulares, desencadenando la inflamación del tejido. Además, se analizaron las declaraciones de 386 pacientes dentales y la aparición de alergias a los antibióticos se informó de un 6,2\% de los pacientes. Las reacciones a las penicilinas fueron predominantes $(4,7 \%)$, seguido de sulfanilamida $(1,0 \%)$ y las cefalosporinas $(0,5 \%)$. Los síntomas predominantes fueron esencialmente cutáneos. También se analizaron las consecuencias, diagnóstico y prevención de las alergias a los antibióticos. Este estudio encontró que los $\beta$-lactámicos fueron los fármacos más alergénicos y la gran mayoría de las reacciones inmunes se desataron en contra ellos. El tratamiento de desensibilización debe considerarse el último enfoque clínico, debido a sus riesgos a los pacientes.

Descriptores: Hipersensibilidad a las Drogas; Antibacterianos; Alergia e Inmunología. 


\section{INTRODUÇÃO}

As reações de hipersensibilidade aos antibióticos e antimicrobianos são condições graves e, por vezes, possuem impacto significativo nos padrões de prescrições desses fármacos ${ }^{1,2}$. Tais reações englobam todos os tipos de reações adversas causadas pela resposta imunológica do paciente a essas drogas, destacando-se a hipersensibilidade imediata, caracterizada pelas respostas denominadas "imediatas e aceleradas", associadas à produção de imunoglobulinas, principalmente da classe $\operatorname{IgE}^{3,4}$, e hipersensibilidade tardia, onde a resposta aos antígenos envolve a resposta imune celular ${ }^{1,5}$.

O tempo decorrido do contato com o antígeno até $o$ desencadeamento da reação de hipersensibilidade não deve ser o único critério para a identificação do tipo de fenômeno envolvido. Entretanto, se apenas o fator "tempo pós-contato" for considerado, alguns casos de hipersensibilidade que ocorrem após as 72 horas podem ainda envolver a presença de $\operatorname{IgE}$ e $\mathrm{IgG}$, sendo que apenas nos casos mais graves de respostas tardias, retardadas, como ocorre com a anemia hemolítica, neutropenia, disfunções renais graves, trombocitopenia, doença do soro, vasculite e infiltração pulmonar, a IgE não está envolvida, mas mesmo nessas condições, outras imunoglobulinas podem ter papel essencial, além da própria resposta celular ${ }^{3}$.

De uma forma geral, os antibióticos e quimioterápicos com atividade antimicrobiana estão entre os fármacos que mais frequentemente induzem reações de hipersensibilidade, destacando-se os $\beta$ lactâmicos pela ocorrência de reações anafiláticas e pela elevada frequência com que reações de hipersensibilidade com manifestações cutâneas são detectadas, principalmente com a exacerbação de seu uso $^{6}$.

Dentre os fatores intrínsecos aos agentes químicos, para o desenvolvimento de uma reação de hipersensibilidade, destaca-se a sua reatividade química e peso molecular ${ }^{7}$. Alguns indivíduos podem desenvolver uma reatividade cruzada, onde anticorpos e células imunorreativas, específicas para uma droga, podem se ligar outros tipos de fármacos do mesmo grupo ou classe química, em função de semelhança estrutural, o que não impede que um mesmo paciente produza anticorpos reativos frente a diversas drogas de um mesmo grupo, como ocorre com os $\beta$-lactâmicos ${ }^{4}$, as drogas mais utilizadas na área médica $\mathrm{e}$ odontológica.

Agentes químicos e/ou biológicos com maior peso molecular são naturalmente imunogênicos para uma parcela significativa da população ${ }^{7}$, mesmo sem sofrer qualquer tipo de modificação, posto que são diretamente reconhecidos pelas células imunocompetentes e podem sensibilizar diretamente o hospedeiro com predisposição genética e ambiental ${ }^{7,8}$. Por outro lado, a maioria dos fármacos com atividade antimicrobiana consiste de compostos de baixo peso molecular que precisam sofrer um processo de haptenização para adquirirem imunogenicidade, após ligação a proteínas carreadoras ${ }^{9}$, como ocorre com os $\beta$-lactâmicos, as drogas mais associadas a quadros de hipersensibilidade $^{10}$.

Nesse processo ocorre a transformação ou hidrólise da droga, formando haptenos de forma direta ou indireta, que se ligam a proteínas sanguíneas e/ou teciduais formando o complexo hapteno-proteína, que apresenta a capacidade de induzir respostas específicas pelo sistema imunológico a partir da atividade de células apresentadoras de antígenos e dos linfócitos $\mathrm{T}_{\mathrm{aux}} 11$.

Embora raras, as reações de hipersensibilidade mais graves e estudadas são do tipo anafilático, que resultam pela formação de $\operatorname{IgE}$ contra o antibiótico e/ou seus derivados metabólicos, produzindo reações anafiláticas que podem evoluir para o óbito em $0,0015 \%$ a $0,002 \%{ }^{10}$. Embora raras, somente as reações anafiláticas às penicilinas representam $75 \%$ dos casos de óbito por hipersensibilidade a drogas nos Estados Unidos ${ }^{12}$. As moléculas de imunoglobulinas produzidas após a sensibilização do hospedeiro pelo par hapteno-proteína carreadora acabam por se ligar a receptores da porção constante $(\mathrm{Fc})$ situados na superfície de mastócitos, onde, em um novo contato, ou mesmo no final do contato inicial, imunogênico, a ligação do antígeno com as moléculas de $\operatorname{IgE}$ na superfície celular irá levar à degranulação de basófilos e mastócitos, com a liberação de grandes quantidades de mediadores químicos ligados aos fenômenos inflamatórios, particularmente a histamina, gerando um quadro cardiovascular e respiratório grave e, não raro, sequelas graves ou mesmo o óbito ${ }^{13}$.

Conquanto ocupando um destaque menos significativo na relevância dada por clínicos, mas de ocorrência bastante comum, as reações de hipersensibilidade tardia têm uma participação nas alergias a drogas antimicrobianas e são essencialmente mediadas pelos linfócitos $\mathrm{T}_{\text {aux }}$.

Embora os mecanismos efetores desses fenômenos alérgicos, incluindo a hipersensibilidade por complexos imunes e citotóxica, já se apresentem descritos, a importância dessas condições na área da saúde requer avaliações constantes, uma vez que sua prevenção e o diagnóstico dos seus primeiros sinais é a forma mais segura de evitar complicações e minimizar quaisquer danos aos pacientes ${ }^{1,9,14}$. Como agravante, muitos profissionais negligenciam a anamnese dos seus pacientes, aumentando o risco de acidentes envolvendo a hipersensibilidade a fármacos. Assim, considerando-se a relevância das reações de 
hipersensibilidade a antibióticos, o objetivo desse estudo foi realizar uma revisão bibliográfica sobre esse fenômeno, apresentar as bases biológicas envolvidas, bem como verificar a ocorrência dessa condição em uma população adulta, com uma abordagem prática.

\section{MATERIAL E MÉTODO}

\section{○ REVISÃO DA LITERATURA}

Esse estudo consiste de revisão da literatura e avaliação de prontuários junto á Faculdade de Odontologia de Araçatuba-UNESP, com análise qualitativa dos dados obtidos.

A revisão da literatura foi realizada a partir das bases de dados indexadas SciELO (Scientific Electronic Library Online) e PubMed (National Library of Medicine). Optou-se por avaliar a literatura entre 1993 e 2015 . Foram associados "drug hypersensitivity", "anti-bacterial drugs", "antibacterial agents", e "allergy and immunology", em inglês, bem como "hipersensibilidade a drogas", "antibacterianos" e "alergia e imunologia", em português, como descritores para o levantamento. Através dos descritores acima, desconsiderando-se os casos de duplicidade, obteve-se um total de 1.785 artigos.

Destes, foram selecionados os artigos que abordavam o mecanismo de envolvidos nas reações de hipersensibilidade aos antibióticos utilizados na clínica médica e, principalmente, odontológica, bem como aqueles que discutiam os aspectos obtidos com a pesquisa de dados em fichas clínicas dos pacientes, obtendo-se 36 artigos.

○ OCORRÊNCIA DE HIPERSENSIBILIDADE A ANTIBIÓTICOS E QUIMIOTERÁPICOS COM ATIVIDADE ANTIMICROBIANA

Como uma indicação para determinar a extensão da ocorrência de fenômenos de hipersensibilidade a fármacos com atividade antibiótica entre pacientes odontológicos que apresentaram infecções bacterianas de cabeça e pescoço, os dados de 386 pacientes dos arquivos do Laboratório de Microbiologia e Imunologia da FOAUNESP foram analisados. Desses pacientes, 124 eram do sexo masculino e 262 eram do sexo feminino, com idade média de 27,4 $\pm 6,1$ anos.

Esses pacientes receberam exames de cultura e antibiograma e/ou participaram de estudos clínicomicrobiológicos junto ao laboratório, entre 1993 e 2014. Dentre as características avaliadas estava gênero, idade, profissão, enfermidade bucal, condição de saúde sistêmica, classe farmacológica do alérgeno e manifestações básicas da alergia, cujos dados eram transferidas para planilhas de contingenciamento e submetidas à análise.
Os testes de Qui-Quadrado, teste exato de Fisher e teste de Mann-Whitney foram utilizados para avaliar a significância das associações entre as variáveis clinicas, com níveis de significância de $5 \%$.

\section{RESULTADOS E DISCUSSÃO}

Os resultados mostraram que $6,2 \%$ dos pacientes declararam apresentar alguma reação de hipersensibilidade a drogas antimicrobianas, sendo que a reação às penicilinas foi observada em $4,7 \%$ dos indivíduos, enquanto que reações às sulfas $(1,0 \%)$ e às cefalosporinas $(0,5 \%)$ foram também observadas. Os dados desses pacientes não evidenciaram hipersensibilidade cruzada com outros fármacos, tampouco reações de hipersensibilidade a outras drogas antimicrobianas.

Quando consideradas isoladamente, nenhum agente mostrou induzir maior ocorrência de reações adversas imunomediadas, tampouco mostrou correlação com idade, condição de saúde sistêmica ou bucal, no momento que a anamnese foi realizada ( $p>0.05)$. Embora a ocorrência de fenômenos de hipersensibilidade tenha se mostrado mais proeminente entre mulheres, com proporção de 2:1 em relação ao sexo masculino como também evidenciado na literatura ${ }^{15}$, essa significância desaparece quando se verifica que essa também foi a distribuição dos pacientes estudados, com uma participação mais elevada de pacientes do sexo feminino. Considerados como grupo, as reações de hipersensibilidade aos agentes antimicrobianos representaram $43 \%$ das declarações de alergia dos pacientes.

$\mathrm{Na}$ análise dos dados outrora coletados na anamnese dos pacientes, observou-se que a ampla maioria das manifestações clínicas da hipersensibilidade se apresentou de uma hora a 72 horas após o contato com o agente antimicrobiano, produzindo urticaria, angioedema, conjuntivite e rinite em $54,2 \%$, enquanto as reações tardias, que ocorrem após 72 horas de contato, com sintomas essencialmente cutâneos, como urticaria, erupções maculopapulares e vasculite, foram relatadas por $41,7 \%$ dos pacientes alérgicos. A ocorrência de reações imediatas com colapso cardiovascular foi denunciada por $4,2 \%$ dos pacientes.

A utilização de dados de prontuários clínicos e/ou laboratoriais, na verificação da frequência e natureza das reações alérgicas na população é problemática, em função da confiabilidade dos dados fornecidos pelos pacientes, mesmo quando adequadamente obtidos ${ }^{1}$, de forma que as informações aqui apresentadas podem estar superestimados, como também relatado na literatura ${ }^{9,16}$, em função da natureza declaratória dos processo. Quando testes de hipersensibilidade são realizados, esses valores são 
significativamente menores ${ }^{16}$.

A despeito de alguns ensaios mostrando baixos níveis de hipersensibilidade aos $\beta$-lactâmicos ${ }^{17}$, a maioria dos estudos relata valores entre $6 \%$ a $10 \%$ como a ocorrência de algum tipo de hipersensibilidade a essa classe de drogas ${ }^{8,15,18}$, onde de $1 \%$ a $3 \%$ dos pacientes expostos apresentam alergia às cefalosporinas e até $10 \%$ dos indivíduos mostram reatividade frente às penicilinas ${ }^{14,19}$, fazendo com que até $2 \%$ das prescrições administradas originem reações imunológicas indesejadas ${ }^{19}$. Essa frequência corresponde a valores superiores aos observados no presente ensaio. Entretanto, dada a natureza da avaliação, muitos pacientes com relatos de ocorrência de reações adversas semelhantes às alergias por antibióticos possivelmente não apresentaram as bases imunológicas do fenômeno, sugerindo tratar-se de efeito direto dos agentes antimicrobianos e não reações de hipersensibilidade, como já relatado ${ }^{16}$.

Dentre as drogas antimicrobianas com maior ocorrência de reações de hipersensibilidade destacamse os $\beta$-lactâmicos, que também constituem o grupo de fármacos mais frequentemente empregados na clínica médica e odontológica, em função de sua eficácia sobre membros da microbiota, baixo custo e relativa segurança, com poucos efeitos colaterais ${ }^{13,14}$. Como a maioria dos antibióticos, os $\beta$-lactâmicos são quimicamente pouco reativos no seu estado original e apresentam um peso molecular pequeno para atuarem como antígenos completos, dotados de imunogenicidade ${ }^{9}$. No entanto, tais agentes se ligam covalentemente aos resíduos de aminoácidos das proteínas séricas ou teciduais, sendo reconhecidos como antígenos pelas células do sistema imunológico ${ }^{9,20}$.

No caso das drogas para as quais se observaram relatos de reações de hipersensibilidade, as penicilinas acabam se ligando aos resíduos de lisina nas proteínas séricas, principalmente a albumina, e teciduais. Processo análogo, mas menos eficiente também ocorre com as cefalosporinas e demais drogas desse grupo, como os carbapenêmicos, monobactâmicos e inibidores de $\beta$-lactamases, justificando a maior ocorrência de reações alérgicas aos $\beta$-lactâmicos ${ }^{9,20}$, cujo anel $\beta$-lactâmico pode se abrir e se ligar covalentemente. Outros fármacos, como as sulfas, tornam-se reativos após a modificação da droga pela ação da enzima P450, que libera um intermediário capaz modificar o grupo tiol das proteínas do hospedeiro 9 .

As penicilinas e demais drogas do grupo originam dois tipos de metabólitos, um considerado principal, o grupo peniciloil ou similar ${ }^{13}$, que é responsável por aproximadamente $95 \%$ das ligações das penicilinas às proteínas carreadoras, e os determinantes secundários, como o peniciloato. Uma das principais diferenças entre os $\beta$-lactâmicos e os outros tipos de antibióticos é que eles sofrem degradação espontânea em condições fisiológicas, facilitando as interações químicas, sendo que a maioria dos outros antimicrobianos sofre metabolismo essencialmente enzimático ${ }^{20}$.

As principais reações de hipersensibilidade aos $\beta$-lactâmicos envolvem a resposta imune humoral, produzindo quadros que tendem a ser imediatos ou acelerados, de uma a 72 horas após o contato ${ }^{2,21}$. Essas reações humorais geralmente envolvem a produção de $\mathrm{IgE}^{22}$, embora quadros associados à $\mathrm{IgG}$ também ocorram, principalmente quando observa-se envolvimento do sistema complemento e formação de imunocomplexos. Clinicamente, observam-se severos fenômenos cutâneos, cardiovasculares, respiratórios, e por vezes, hematológicos, como na anemia hemolítica, causada pela destruição de hemácias devido à ligação de imunoglobulinas e complemento na superfície da membrana de celular, onde se encontram as moléculas do fármaco ${ }^{13}$. Nesse último caso, os danos não ocorrem de forma tão rápida, podendo demorar dias para se manifestarem clinicamente ${ }^{23}$. Os pacientes também desenvolvem quadro de hipertermia medicamentosa pela liberação de IL-1, que tende a regredir com a interrupção do contato com a droga.

A via e frequência da administração do agente antimicrobiano podem ter influência no desenvolvimento das reações de hipersensibilidade, sendo que a via oral é considerada a menos sensibilizante. No presente ensaio, não foi possível estabelecer uma correlação entre a ocorrência de reações de hipersensibilidade e a via de administração da droga. A idade é outro fator diretamente relacionado com a frequência dessas reações, onde as crianças estão particularmente expostas a quadros severos em função do uso de antibióticos para infecções respiratórias bacterianas, além de infecções virais concomitantes, que aumentam a liberação de interferon e a apresentação de antígenos aos linfócitos $\mathrm{T}_{\text {aux, }}$ exacerbando a reação imunológica aos alérgenos ${ }^{20}$. Entretanto, entre os adultos, os portadores de hipersensibilidade a drogas, em particular os $\beta$ lactâmicos, apresentam idade média maior do que os pacientes não reativos ${ }^{9}$.

Fatores hereditários podem modificar a expressão da alergia aos fármacos ${ }^{24}$, porém são desconhecidos os mecanismos através dos quais esse fenômeno se dá ${ }^{20,24}$, não tendo sido detectadas diferenças significativas na distribuição de polimorfismos gênicos associados à expressão de interleucinas e seus receptores em pacientes com hipersensibilidade a antibióticos ${ }^{25}$, tampouco nos padrões genéticos do HLA (complexo de antígenos leucocitários humanos) e genes ligados ao complexo de histocompatibilidade principal humano ${ }^{8}$. 
Dados iniciais sugerem que a hipersensibilidade às penicilinas tem bases poligênicas, o que pode explicar porque pacientes com atópicos para essa classe de drogas também reagem com outros fármacos que não possuem semelhança estrutural com os $\beta$ lactâmicos. Dentre os genes associados às reações de hipersensibilidade aos $\beta$-lactâmicos destaca-se o controle da expressão da IL-4, que está envolvido na regulação da hipersensibilidade imediata $^{8}$. Dessa forma, devido a essa natureza poligênica da alergia aos $\beta$-lactâmicos, onde podem ocorrer reações de hipersensibilidade severas mesmo a drogas que não são estruturalmente relacionadas às penicilinas, o grau de segurança da utilização desses antibióticos para indivíduos com alergia a penicilina é questionável ${ }^{8}$.

Uma vez que a reação imunológica frente aos $\beta$ lactâmicos é voltada primeiramente para o anel $\beta$ lactâmico, a reatividade imunológica cruzada é uma realidade frequente ${ }^{3,26-28}$, embora a redução da utilização dos fármacos naturais, como a penicilina $\mathrm{G}$, tenha reduzido a frequência de reação ao anel característico e esteja produzindo uma exacerbação das reações alérgicas às cadeias laterais e demais radicais nas novas drogas 3,27 .

De 5 a $10 \%$ dos pacientes alérgicos às penicilinas apresentam essa reatividade cruzada com as cefalosporinas ${ }^{3}$, mas para as drogas de segunda e terceira geração não apresentam valores tão elevados ${ }^{18}$. No presente estudo, não foram observados pacientes que relatassem alergia a penicilinas $\mathrm{e}$ cefalosporinas, mas pela natureza da investigação realizada, não se pode excluir essa possibilidade.

Inicialmente acreditava-se que a reatividade cruzada entre os compostos dessa classe dependia unicamente da porção da molécula reconhecida como antígeno e devido à semelhança estrutural entre os fármacos, o paciente com hipersensibilidade a um agente também deveria sê-lo aos demais, o que não é uma realidade, visto que atualmente se reconhece que mais importante do que o anel cíclico típico do grupo, as cadeias laterais e os radicais são os principais alvos das reações de hipersensibilidade ${ }^{27}$. Nesses casos, de a alergia voltada contra a cadeia lateral da molécula, o paciente poderá entrar em contato com outros tipos de $\beta$-lactâmicos sem acarretar, na maioria das vezes, complicações $^{4,27}$. Diante da importância do fenômeno e reforçando a necessidade de segurança clínica para a prescrição de drogas, a seleção do fármaco deverá ser realizada caso a caso, buscando alternativas que não compartilhem do histórico narrado pelo paciente, principalmente quando o fenômeno de hipersensibilidade descrito for relativo às penicilinas, as mais alergênicas ${ }^{27}$.

Comparando as cefalosporinas de primeira, segunda e terceira geração, observa-se uma maior frequência de reatividade cruzada relacionada com as duas primeiras, devido à menor imunogenicidade das cadeias laterais das últimas. A elevada alta reatividade cruzada in vitro entre penicilinas e cefalosporinas não é a observada "in vivo" 29 . Assim, quando há a necessidade de administrar cefalosporinas a um paciente com histórico de hipersensibilidade às penicilinas, é preciso realizar, previamente testes cutâneos, e o tratamento pode ser iniciado, caso o resultado seja negativo, pois indica que o paciente é reativo apenas à cadeia lateral do antibiótico ${ }^{30}$. Uma exceção a essa reatividade são o monobactâmicos, como o aztreonam, que, pela ausência do núcleo bicíclico, não apresentam reatividade com penicilina e são opção terapêutica para esses $\operatorname{casos}^{4,29}$.

A ocorrência de reações alérgicas às sulfas varia de $0,2 \%$ a $4 \%$ dos pacientes imunologicamente competentes $^{9,31}$, exacerbando-se significativamente em pacientes com desordens no sistema de defesa ${ }^{31}$, com predomínio das reações aceleradas e tardias mais leves, sem reações cruzadas com drogas não pertencentes ao grupo da sulfanilamida ${ }^{9}$. Deve-se ressaltar que essa ocorrência é, proporcionalmente ao uso que se dá a esse grupo de drogas, bastante elevado $^{32}$

No presente ensaio, $1 \%$ dos indivíduos relatou a ocorrência de reações às sulfas, que caracterizam-se, quase sempre, por erupções maculopapulares e eritema multiforme, mas pode produzir quadros como febre, necrólise epidermal aguda urticária, angioedema, lesões pustulosas exantemáticas agudas, fotossensibilidade, dermatite esfoliativa e anafilaxia ${ }^{32}$.

De uma forma geral, a melhor abordagem para evitar reações de hipersensibilidade em pacientes com esse histórico é evitar a prescrição de medicamentos que pertençam ao mesmo grupo do fármaco que produziu a reação notificada ${ }^{32}$, o que nem sempre é possível, como ocorre em pacientes HIV positivos com hipersensibilidade às sulfas e que necessitam dessa classe de drogas (amprenavir e fosamprenavir) como inibidores de protease ${ }^{32}$. Nessas situações, podese optar pela terapia de dessensibilização, que apresenta diversas limitações em termos de efetividade e segurança ${ }^{33}$.

Aspecto relevante observado entre os dados coletados foi a ausência de relatos de alergias aos macrolídeos e lincosaminas, drogas amplamente utilizadas na clínica médico-odontológica como opção viável aos pacientes com histórico de hipersensibilidade aos $\beta$-lactâmicos. Os macrolídeos sofrem a ação de enzimas do complexo citocromo p450, por desmetilação ${ }^{34}$, originando haptenos capazes de induzir reações alérgicas quando associados a proteínas carreadoras.

De uma forma geral, para os macrolídeos, as reações adversas associadas a esses fármacos são suaves em intensidade e raras em ocorrência ${ }^{34}$, o que 
associado à elevada sensibilidade da maioria dos membros da microbiota autóctone humana e patógenos clássicos e patógenos respiratórios faz com que seu uso venha se generalizando ${ }^{35}$.

Como mencionado acima, nenhum dos pacientes com história de hipersensibilidade aos antibióticos e quimioterápicos com atividade antimicrobiana relataram reações ao macrolídeos e lincosaminas, sendo que a raridade das reações a essas drogas é atestada na literatura ${ }^{5}$, acometendo apenas $0,4 \%$ a $3 \%$ dos tratamentos instituídos ${ }^{9}$. Casos raros de reações imediatas, na forma de anafilaxia, e reações não imediatas, como erupções cutâneas, necrose epidérmica tóxica e vasculite leucocitoclástica foram relatadas para claritromicina e azitromicina ${ }^{5}$.

Para as lincosaminas, a ocorrência de reações alérgicas mais severas e imediatas, como a anafilaxia, é bastante incomum ${ }^{6}$, embora os efeitos colaterais relativos à supressão da microbiota normal constitua um elemento a ser considerado quando sua prescrição de fizer necessária. Por outro lado, a dessensibilização tem sido relatada como bem-sucedida ${ }^{5}$, mas as diversas complicações dessa abordagem somente se justificam diante da necessidade imperiosa da utilização dessas drogas e absoluta ausência de outra opção terapêutica ${ }^{36}$.

Inicialmente o diagnóstico das reações de hipersensibilidade aos antimicrobianos é clínico, mas reações dessa natureza podem se assemelhar a muitas condições clínicas, de forma que o diagnóstico definitivo depende de testes cutâneos, de provocação, e por contato, além de ensaios laboratoriais que quantifiquem o total de $\operatorname{IgE}$ e a proporção dessas imunoglobulinas que reagem com os fármacos que desencadearam a reação ${ }^{22}$. Os testes cutâneos e similares acabam identificando a presença da resposta humoral a um determinado antibiótico, mas é disponível apenas para alguns fármacos, $\beta$-lactâmicos em particular. Por fim os testes por contato são utilizados para reações de hipersensibilidade a medicamentos que envolvem mecanismos mediados por células $\mathrm{T}_{\mathrm{aux}}$.

Para os pacientes alérgicos, deve-se optar por minimizar o contato com o fármaco com o qual o histórico de alergia foi contruído. Entretanto, nem sempre essa é uma condição possível, podendo-se partir para a dessensibilização do pacientes, mas a eficiência desse processo é significativa apenas quando o quadro de hipsersensibilidade está ligado à produção de $\operatorname{IgE}^{36}$. Existem vários protocolos com esse objetivo, que defendem a administração soluções contento o alérgeno em concentrações diminutas, mas crescentes, produzindo aquilo que Legendre et al. ${ }^{36}$ (2014) descreveram como uma "anafilaxia controlada", onde mastócitos ligados à $\operatorname{IgE}$ degranularão em concentrações modestas do alérgeno e não darão uma resposta sistêmica. $\mathrm{Na}$ medida em que as dosagens do fármaco forem sendo duplicadas, o que geralmente é feito a cada 15 minutos, obsrva-se a supressão dos receptores de IgE na superfície da mastócitos e quando isso ocorre, a dose clínica necessária da droga poderá ser utilizada ${ }^{30,36}$.

É importante enfatizar que essa modalidade de dessensibilização perdura por um período de 24-36 horas, nas quais os mastócitos não mais degranulam em função da supressão induzida, mas o paciente permanece com as bases imunológicas de sua hipersensibilidade ao antimicrobiano e poderá ter uma reação intensa à droga fora desse período de segurança ${ }^{10}$. Como as terapias de dessensibilização podem, elas próprias dar origem a quadros de hipersensibilidade graves, as mesmas não são indicadas para pacientes com histórico de síndrome de Stevens-Johnson, necrólise epidermal tóxica, quadros de vasculites, citopenias e reações sistêmicas ${ }^{33}$.

\section{CONCLUSÃO}

Os resultados desse levantamento bibliográfico e análise de dados de pacientes evidenciaram que as penicilinas representam $75,8 \%$ dos casos de pacientes com histórico de hipersensibilidade a drogas, enquanto que os $\beta$-lactâmicos, como um todo, são responsáveis por $83,9 \%$ dos quadros alérgicos, enquanto as sulfas compreendem $16,1 \%$ desses quadros. A grande diversidade de mecanismos envolvidos nessas reações e suas manifestações sugerem que a escolha da droga para indivíduos hipersensíveis deve evitar fármacos estruturalmente relacionados com o alérgeno e a terapia de dessensibilização seja utilizada apenas quando outras possibilidades não puderem ser implementadas.

\section{REFERÊNCIAS}

1. Chiriac AM, Demoly P. Drug allergy diagnosis. Immunol Allergy Clin N Am. 2014;34:461-71.

2. Doña I, Barrionuevo E, Blanca-Lopez N, Torres MJ, Fernandez TD, Mayorga $\mathrm{C}$ et al. Trends in hypersensitivity drug reactions: more drugs, more response patterns, more heterogeneity. J Investig Allergol Clin Immunol. 2014;24:143-53.

3. Yates AB. Management of patients with a history of allergy to beta-lactam antibiotics. Am J Med. 2008;121:572-6.

4. Gaeta F, Valluzzi R L, Alonzi C, Maggioletti M, Caruso C, Romano A. Tolerability of aztreonam and carbapenems in patients with IgE-mediated hypersensitivity to penicillins. J Allergy Clin Immunol. 2015;135:972-6.

5. Bernard YHT. Update on the management of antibiotic allergy. Allergy Asthma Immunol. Res. 2010;2:77-86. 
6. Bulloch NM, Baccas JT, Arnold S. Clindamycininduced hypersensitivity reaction. Infection 2015. DOI 10.1007/s15010-015-0826-2

7. Faria E. Diagnóstico de alergia a drogas: atualização. Rev. Bras. Alerg. Imunopatol. 2008;31:133-8.

8. Apter AJ , Schelleman H, Walker A, Addya K, Rebbeck T. Clinical and genetic risk factors of self-reported penicillin allergy. J Allergy Clin Immunol. 2008;122:152-8.

9. Romano A, Warrington R. Antibiotic allergy. Immunol Allergy Clin N Am. 2014;34:489-506.

10. Mirakian R, Leech SC, Krishna MT, Richter AG, Huber PAJ, Farooque $\mathrm{S}$ et al. Management of allergy to penicillins and other beta-lactams. Clin Exp Allergy 2015;45:300-27.

11. Benno S, Werner JP. Mechanisms of drug-induced allergy. Mayo Clin. Proc. 2009;84:268-72.

12. Neugut AI, Ghatak AT, Miller RL. Anaphylaxis in the United States: an investigation into its epidemiology. Arch Intern Med. 2001;161:15-21.

13. BERND LAG. Alergia a medicamentos. Rev Bras Alerg Imunopatol. 2005;28:125-32.

14. Macy E. Penicillin and beta-lactam allergy: epidemiology and diagnosis. Curr Allergy Asthma Rep. 2014;14:476. DOI 10.1007/s11882-0140476-y

15. Fernández-Rubio ME, Cuesta-Rodríguez T, Urcelay-Segura, Cortés-Valdés C. Alergia a antibióticos $\beta$-lactámicos en cirugía de cataratas. Prevalencia y características preoperatorias de los pacientes alérgicos. Arch Soc Esp Oftalmol. 2014;89:92-8.

16. Andrade D, Jojima K, Lacerda A, Machado LMO, Ensina LF, Nunes ICC, Sole D. Beta-lactam hypersensitivity: not always what it seems. World Allergy Org J. 2015;8(Supl 1):A168.

17. Salden OAE, Rockmann H, Verheij TJM; Broekhuizen BDL. Diagnosis of allergy against beta-lactams in primary care: prevalence and diagnostic criteria. Fam Pract. 2015;32:257-62.

18. Pichichero ME. A review of evidence supporting the American Academy of Pediatrics recommendation for prescribing cephalosporin antibiotics for penicillin-allergic patients. Pediatrics. 2005;115:1048-57.

19. Apter AJ, Kinman JL, Bilker WB, Herlim M, Margolis DJ Lautenbach E et al. Represcription of penicillin after allergic-like events. J Allergy Clin Immunol. 2004;113:764-70.

20. Solensky R. Hypersensitivity reactions to betalactam antibiotics. Cli Rev Allergy Immunol. 2003;3:201-20.

21. Torres MJ, Romano A, Blanca M, Fernandez J, Weck A, Brockow K et al. Diagnosis of immediate allergic reactions to beta-lactam antibiotics. Allergy. 2003;58:961-72.

22. Vultaggio A, Virgili G, Gaeta F, Romano A, Maggi E, Matucci A. High serum $\beta$-lactams specific/total $\mathrm{IgE}$ ratio is associated with immediate reactions to $\beta$-lactams antibiotics. Plos One 2015. DOI:10.1371/journal.pone.0121857

23. Romano A, Torres MJ, Blanca M, Fernandez J, Weck A, Brockow $\mathrm{K}$ et al. Immediate allergic reactions to beta-lactams: diagnosis and therapy. Int. J. Immunophatol Pharmacol. 2003;16:19-23.

24. Pirmohamed M. Genetic factors in the predisposition to drug-induced hypersensitivity reactions. AAPS J. 2006;8:E20-E26.

25. Li J, Liu X-Y, Li L-J, You C-G, Shi L, Zhang CD. Correlation analysis of gene polymorphisms and $\beta$-lactam allergy. J Zhejiang Univ-Sci B (Biomed \& Biotechnol). 2015;16:632-9.

26. Blanca M, Mayorga C, Torres MJ, Warrington R, Romano A, Demoly $\mathrm{P}$ et al. Side-chain-specific reactions to betalactams: 14 years later. Clin Exp Allergy. 2002;32:192-7.

27. Montañez MI, Ariza A, Mayorga C, Fernandez TD, Torres MJ. Cross-reactivity in beta-lactam allergy: alternative treatments. Curr Treat Options Allergy 2015;2:141-54.

28. Żukiewicz-Sobczak WA, Wróblewska P, Adamczuk P, Zwoliński J, Oniszczuk A, WojtyłaBuciora $\mathrm{P}$ et al. Drugs as important factors causing allergies. Postep Derm Alergol. 2015; XXXII:388-92. DOI: 10.5114/pdia.2014.44021

29. Christopher WJ,Cheryle GT. Cross-reactivity of beta-lactam antibiotics. Proc. Bayl Univ Med Cent. 2001;14:106-7.

30. Rosário NA, Grumach AS. Alergia a betalactâmicos na clínica pediátrica: uma abordagem prática. J Pediatr. 2006;82:181-8.

31. Gruchalla R. Drug allergy. J Allergy Clin Immunol. 2003;111:S548-59.

32. Kuyucu S, Mori F, Atanaskovic-Markovic M, Caubet J-C, Terreehorst I, Gomes E, et al. Hypersensitivity reactions to non-betalactam antibiotics in children: An extensive review. Pediatr Allergy Immunol. 2014;25:534-43.

33. Cernadas JR. Desensitization to antibiotics in children. Pediatr Allergy Immunol. 2013:24:3-9.

34. Araujo L, Demoly P. Macrolides allergy. Curr Pharm Design. 2008; 14:2840-62.

35. Guimarães DO, Momesso LS, Pupo MT. Antibióticos: Importância terapêutica e perspectivas para a descoberta e desenvolvimento de novos agentes. Quim. Nova. 2010;33:667-79.

36. Legendre DP, Muzny CA, Marshall GD, Swiatlo E. Antibiotic hypersensitivity reactions and approaches to desensitization. Clin Practice 2014;58:1140-8. 


\section{CONFLITO DE INTERESSES}

Os autores declaram não haver conflitos de interesse.

\section{AUTOR PARA CORRESPONDÊNCIA}

Ellerson Gaetti Jardim Júnior

egaettij@foa.unesp.br

Submetido em 21/01/2016

Aceito em 29/01/2016 